Original Article

\title{
Slow Expiration Reduces External Oblique Muscle Activity during Maximum Trunk Flexion
}

\author{
Hiroshi Ishida, PhD, PT ${ }^{1)^{*}}$, Saya Tajima, PT ${ }^{2)}$, Riyo Masuno, $\mathrm{PT}^{3)}$, Yoshiko Kogame, $\mathrm{PT}^{4}$, \\ Suguru Ando, $\mathrm{PT}^{4}$, Katsuhiko Yokohata, $\mathrm{PT}^{5)}$, Susumu Watanabe, PhD, $\mathrm{PT}^{1)}$ \\ 1) Department of Rehabilitation, Faculty of Health Science and Technology, Kawasaki University of \\ Medical Welfare: 288 Matsushima, Kurashiki, Okayama 701-0193, Japan \\ 2) Department of Rehabilitation, Ishikawa Hospital, Japan \\ 3) Department of Rehabilitation, Mizushima Kyodo Hospital, Japan \\ 4) Department of Physical Therapy, Kurashiki Heisei Hospital, Japan \\ 5) Department of Physical Therapy, Kurashiki Daiichi Hospital, Japan
}

\begin{abstract}
Purpose] The purpose of this study was to demonstrate the effects of expiration on abdominal muscle activity during maximum trunk flexion. [Subjects] Twenty-one healthy university students (10 men, 11 women) participated in this study. [Methods] Electromyography (EMG) was used to quantify the activity of the right rectus abdominis, external oblique, and internal oblique muscles. The paired t-test was used to examine the significance of differences in the abdominal muscles between maximum trunk flexion with breath holding and slow expiration. [Results] There was a significantly lower EMG activity in the external oblique muscle during maximum trunk flexion with slow expiration. [Conclusion] The results of this study indicate that slow expiration reduces external oblique muscle activity during maximum trunk flexion performed by healthy young subjects.

Key words: Expiration, Electromyography, Abdominal muscles
\end{abstract}

(This article was submitted Apr. 28, 2014, and was accepted Jun. 17, 2014)

\section{INTRODUCTION}

Various abdominal exercises are used in physical therapy for the treatment of low back injury and as a component of fitness training programs ${ }^{1-4)}$. Clinicians should be knowledgeable about the types of exercise and training programs that would most effectively recruit abdominal muscles, as frail elderly individuals are unable to exert the effort required for strenuous exercise. The activities of the rectus abdominis (RA), external oblique (EO), and internal oblique (IO) muscles during performance of abdominal exercises in combination with expiration have been investigated because abdominal muscles are the principal muscles involved in forced expiration ${ }^{5,6)}$. Yoon et al. ${ }^{5}$ reported that the activities of the transversus abdominis and IO (TrA/ IO) are significantly greater during an abdominal curl-up with slow expiration than during an abdominal curl-up with quiet inspiration. Ishida and Watanabe ${ }^{6)}$ showed that abdominal muscle activities (RA, EO, and IO) increased when maximum expiration was held in the side bridge exercise compared with resting expiration. These studies used exercises requiring sub-maximal voluntary contraction of

*Corresponding author. Hiroshi Ishida (E-mail: ishida@ mw.kawasaki-m.ac.jp)

(C2014 The Society of Physical Therapy Science. Published by IPEC Inc. This is an open-access article distributed under the terms of the Creative Commons Attribution Non-Commercial No Derivatives (by-ncnd) License $<$ http://creativecommons.org/licenses/by-nc-nd/3.0/>. the abdominal muscles to quantify the additive effects of expiration. The capacity for muscular activity is related to the number of active motor units and their level of activation. If motor units are activated in a task-specific manner, a muscle's maximal activity will differ with the task. It is possible that the highest muscular activity would occur during a dual task condition (maximum trunk flexion with expiration) in which there would be a summation of the activities of two task-specific motor unit populations. To the best of our knowledge, no study has measured the muscle activities of abdominal muscles during maximum trunk flexion with expiration. One of the reasons for selecting the maximum trunk flexion exercise is its ease of performance compared to other abdominal strengthening exercises. The purpose of this study was to demonstrate the effects of expiration on abdominal muscle activity during maximum trunk flexion.

\section{SUBJECTS AND METHODS}

Twenty-one healthy university students (10 men, 11 women) participated in this study. Their age, height, and weight (mean $\pm \mathrm{SD}$ ) were $21.3 \pm 2.0$ years, $164.6 \pm 8.4 \mathrm{~cm}$, and $59.5 \pm 12.2 \mathrm{~kg}$, respectively. The study was explained to the subjects, whose consent was obtained prior to their participation. The protocol for this study was approved by the Ethics Committee of the Kawasaki University of Medical Welfare.

Electromyography (EMG) was conducted using the MQ8 system (Kissei Comtec, Japan). Skin preparation of the electrode sites involved cleansing with alcohol. Disposable 
silver/silver chloride surface electrodes with a recording diameter of $1 \mathrm{~cm}$ (Blue Sensor P-00-S, Ambu, Denmark) were used. The EMG signals of the right RA, EO, and TrA/ IO muscles were recorded. Electrode placement was based on previous work that described the following positions for these muscles ${ }^{5,6)}$ : RA, electrode positioned $2.5 \mathrm{~cm}$ lateral to the umbilicus; EO, electrode positioned at the anterior end of the eighth rib; and $\mathrm{TrA} / \mathrm{IO}$, electrode positioned horizontally within a triangle consisting of the inferior border made up of the line from the anterior-superior iliac spine (ASIS) to the pubic symphysis, and the superior border made up of the line from one ASIS to the other. Bipolar electrode pairs were placed longitudinally over the muscle at $2.5-\mathrm{cm}$ intervals. A ground electrode was placed over the ASIS.

Wide straps secured the thorax, pelvis, and thighs during maximum voluntary trunk flexion in the supine position (Fig. 1). Subjects were asked to hold maximum trunk flexion for $5 \mathrm{~s}$. During trunk flexion the following 2 respiration patterns were performed: (1) holding the breath after resting expiration with an open airway (breath holding); and (2) breathing out from the end of resting expiration to the maximum expiration level over $3 \mathrm{~s}$ in the middle of the task (slow expiration). Subjects were allowed to practice until they could perform the tasks consistently. While the subjects performed each task, EMG signals were measured for $3 \mathrm{~s}$ in the middle of the task. The 2 tasks were performed in a random order. Data were collected 3 times for each task. The EMG signals were amplified, digitized at a sampling frequency of $1,000 \mathrm{~Hz}$, band-pass filtered $(10-500 \mathrm{~Hz})$, and full-wave rectified. The mean muscle activity values over the 3 -s sample of the 3 trials performed for each of the 2 tasks were averaged and used in the statistical analysis. IBM SPSS Statistics 22 was used for all statistical analyses. The paired t-test was used to examine the significance of differences in the abdominal muscle activities between maximum trunk flexion with breath holding and slow expiration. Differences were considered statistically significant at values of $\mathrm{p}<0.05$.

\section{RESULTS}

The mean muscle activity values are listed in Table 1 . The EO muscle showed a significantly lower muscle activity during maximum trunk flexion with slow expiration than during breath holding. The RA and TrA/IO muscle activities were not significantly different between the tasks.

\section{DISCUSSION}

To the best of our knowledge, this study is the first to investigate the effects of performing a maximum trunk flexion exercise with expiration on EMG activity of the abdominal muscles. Our results show that maximum trunk flexion combined with slow expiration did not change the RA and TrA/IO activities compared to breath holding. Previous studies have shown that expiration activates abdominal muscles during trunk exercises (side bridge exercise and abdominal curl-up exercise) ${ }^{5,6}$. Side bridge exercise elicited $17.6 \%$ maximum voluntary contraction (MVC) in the RA, $26.9 \% \mathrm{MVC}$ in the EO, and $20.3 \% \mathrm{MVC}$ in the $\mathrm{IO}^{6}$.

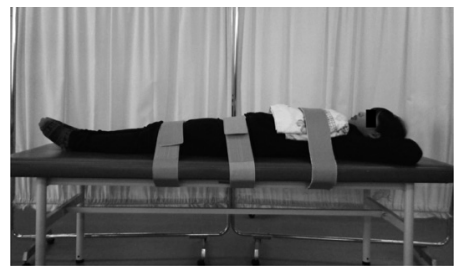

Fig. 1. Start posture of measurement

Table 1. The mean \pm SD values of the abdominal muscle activity

\begin{tabular}{ccc}
\hline & Breath holding $(\mathrm{mV})$ & Slow expiration $(\mathrm{mV})$ \\
\hline RA & $0.15 \pm 0.11$ & $0.14 \pm 0.11$ \\
EO & $0.08 \pm 0.05$ & $0.06 \pm 0.04^{*}$ \\
$\mathrm{TrA} / \mathrm{IO}$ & $0.11 \pm 0.07$ & $0.10 \pm 0.08$ \\
\hline
\end{tabular}

RA: rectus abdominis. EO: external oblique. TrA/IO: transversus abdominis and internal oblique. $*: \mathrm{p}<0.05$

To classify low and high muscle activities, a previous study categorized $0 \%$ to $20 \% \mathrm{MVC}$ as low muscle activity, $21 \%$ to $40 \% \mathrm{MVC}$ as moderate muscle activity, $41 \%$ to $60 \% \mathrm{MVC}$ as high muscle activity, and greater than $60 \%$ MVC as very high muscle activity ${ }^{7}$. The difference between the results of our study and those of other studies might be related to the intensity of the tasks, since a very high-load abdominal exercise was used in this study, compared to low to moderate-load abdominal exercises in previous studies. In this study, the difficulty in augmentation of muscle activities in healthy young subjects, who were capable of exerting effort during exercise requiring exertion, may be due to the activity level of the abdominal muscles during maximum trunk flexion being too high to recruit more motor units during slow expiration.

In this study, maximum trunk flexion with slow expiration significantly reduced only the EO activity among the abdominal muscles. Efferent synergistic inhibition among abdominal muscles is a possible explanation for this decrease. When a subject tries to move a body part, excitation of the cortical units responsible for the muscles moving that part is accompanied by the inhibition of the cortical units responsible for the neighboring muscles ${ }^{8}$. Because the RA is the principal muscle of trunk flexion and the deep abdominal muscles are principal muscles of slow expiration ${ }^{1,9,10)}$, the EO activity might be inhibited. The limitation of this study lies in the inclusion of only healthy young subjects in the analysis. As part of the normal aging process, there is an overall decline in skeletal muscle mass and strength, and the elderly are unable to make the necessary effort during exercise requiring exertion. Further investigation is necessary to determine whether expiration changes the abdominal muscle activity during maximum trunk flexion exercise in the elderly. Future studies should also investigate more than one exercise to examine abdominal muscle activity and to generalize the findings. 


\section{REFERENCES}

1) Axler CT, McGill SM: Low back loads over a variety of abdominal exercises: searching for the safest abdominal challenge. Med Sci Sports Exerc, 1997, 29: 804-811. [Medline] [CrossRef]

2) Juker D, McGill S, Kropf P, et al.: Quantitative intramuscular myoelectric activity of lumbar portions of psoas and the abdominal wall during a wide variety of tasks. Med Sci Sports Exerc, 1998, 30: 301-310. [Medline] [CrossRef]

3) Kavcic N, Grenier S, McGill SM: Quantifying tissue loads and spine stability while performing commonly prescribed low back stabilization exercises. Spine, 2004, 29: 2319-2329. [Medline] [CrossRef]

4) Okubo $Y$, Kaneoka K, Imai A, et al.: Electromyographic analysis of transversus abdominis and lumbar multifidus using wire electrodes during lumbar stabilization exercises. J Orthop Sports Phys Ther, 2010, 40: 743-750. [Medline] [CrossRef]

5) Yoon TL, Kim KS, Cynn HS: Slow expiration reduces sternocleidomastoid activity and increases transversus abdominis and internal oblique muscle activity during abdominal curl-up. J Electromyogr Kinesiol, 2014, 24 228-232. [Medline] [CrossRef]

6) Ishida $\mathrm{H}$, Watanabe $\mathrm{S}$ : Maximum expiration activates the abdominal muscles during side bridge exercise. J Back Musculoskeletal Rehabil, 2014, (in press).

7) Escamilla RF, Lewis C, Bell D, et al.: Core muscle activation during Swiss ball and traditional abdominal exercises. J Orthop Sports Phys Ther, 2010, 40: 265-276. [Medline] [CrossRef]

8) Ohtsuki T: Inhibition of individual fingers during grip strength exertion. Ergonomics, 1981, 24: 21-36. [Medline] [CrossRef]

9) Ishida H, Watanabe S: Changes in lateral abdominal muscles' thickness immediately after the abdominal drawing-in maneuver and maximum expiration. J Bodyw Mov Ther, 2013, 17: 254-258. [Medline] [CrossRef]

10) Kaneko H, Sato H, Maruyama H: Evaluation of lateral abdominal muscle activity during expiratory threshold loading by ultrasonography. J Phys Ther Sci, 2006, 18: 187-191. [CrossRef] 